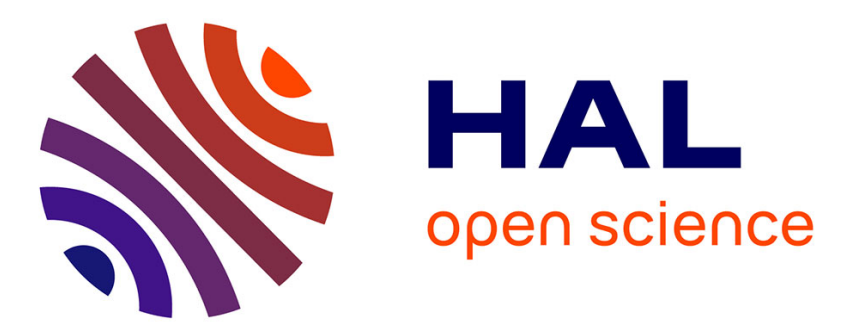

\title{
Conséquences de la forêt méditerranéenne sur les écoulements de crue - Synthèse des recherches menées en France
}

Claude Cosandey, Jacques Lavabre, Claude Martin, Nicolle Mathys

\section{- To cite this version:}

Claude Cosandey, Jacques Lavabre, Claude Martin, Nicolle Mathys. Conséquences de la forêt méditerranéenne sur les écoulements de crue - Synthèse des recherches menées en France. La Houille Blanche

- Revue internationale de l'eau, 2002, 3, pp.38-42. hal-00325288

\section{HAL Id: hal-00325288 \\ https://hal.science/hal-00325288}

Submitted on 27 Sep 2008

HAL is a multi-disciplinary open access archive for the deposit and dissemination of scientific research documents, whether they are published or not. The documents may come from teaching and research institutions in France or abroad, or from public or private research centers.
L'archive ouverte pluridisciplinaire HAL, est destinée au dépôt et à la diffusion de documents scientifiques de niveau recherche, publiés ou non, émanant des établissements d'enseignement et de recherche français ou étrangers, des laboratoires publics ou privés. 


\title{
Conséquences de la forêt méditerranéenne sur les écoulements de crue Synthèse des recherches menées en France
}

\author{
Effects of Mediterranean forest on floods \\ Synthesis of French studies
}

\author{
Claude Cosandey*; Jacques Lavabre $* *$; Claude Martin***; Nicolle Mathys**** \\ * Laboratoire de Géographie Physique, UMR 8591 du CNRS, 1 Place Aristide Briand, F-92190 MEUDON, \\ France-E-mail : cosandey@cnrs-bellevue.fr \\ ** Cemagref, B.P. 31, Le Tholonet, F-13612 AIX-EN-PROVENCE cedex 1, France - E-Mail : \\ jacques.lavabre@cemagref.fr \\ ***UMR 6012 "ESPACE", Equipe GVE, Département de Géographie, 98 Boulevard Edouard Herriot, BP \\ 3209, F-06204 NICE cedex 3, France-E-mail : martincl@infonie.fr \\ ****Cemagref, 2 Rue de la Papeterie, BP 76, 38402 SAINT-MARTIN-D'HERES, France - E-Mail: \\ nicolle.mathys@cemagref.fr
}

\section{Résumé}

Trois ensembles de bassins expérimentaux permettent d'étudier, en France méditerranéenne, les conséquences de la forêt sur les crues. Les résultats diffèrent de façon considérable d'un bassin à l'autre, montrant la complexité des relations pluies-débits. Il est à noter que l'augmentation des crues extrêmes est loin d'être avérée dans tous les cas. Une réflexion sur le fonctionnement hydrologique des bassins permet de lever la contradiction apparente entre les résultats observés.

\begin{abstract}
Three experimental watersheds allow studying hydrological consequences of Mediterranean forest in France. Results are very different from one to other, in relation with complexity of rain-runoff relationship. Forest cut does not systematically increase floods. Considerations about hydrological processes allow understanding apparent contradiction between different results.
\end{abstract}

\section{INTRODUCTION}

Le rôle régulateur de la forêt sur les crues, s'il est largement admis par l'opinion publique, suscite encore bien des interrogations parmi les hydrologues, surtout en ce qui concerne les plus fortes d'entre elles. Il est vrai que ce rôle dépend d'un grand nombre de facteurs, ce qui explique la disparité - lorsque ce n'est pas la divergence - des résultats observés.

Dans le sud de la France, trois sites de BVRE permettent de conduire cette recherche en climat méditerranéen humide. Ces sites diffèrent par leurs conditions pédologiques et géomorphologiques, mais tous sont soumis à de fortes pluies d'automne, et parfois d'été.

La diversité des réponses observées conduit à essayer de dégager les mécanismes qui permettent de rendre compte des conséquences de la végétation sur la formation des écoulements de crue.

\section{SITES D'ETUDE ET CONDITIONS D'EXPERIMENTATION}

Les trois sites pris en compte sont : celui de Draix, situé dans les marnes noires de la région de Digne, dans l'arrière pays provençal; celui du Réal Collobrier, très proche de la côte méditerranéenne; celui du Mont-Lozère enfin, sur les contreforts sud-est du Massif Central (Fig. 1). Les caractéristiques des bassins versants considérés dans cette étude sont portées dans le tableau 1. 
Tableau 1 - Principales caractéristiques des bassins étudiés.

\begin{tabular}{|c|c|c|c|c|}
\hline Nom du bassin & Superficie & Occupation du sol & Pente & Période d'étude \\
\hline $\begin{array}{l}\text { DRAIX } \\
\text { Laval } \\
\text { Brusquet }\end{array}$ & 86 ha & $\begin{array}{c}22 \% \text { bosquets ; } 10 \% \\
\text { herbe ; } 68 \% \text { sols nus }\end{array}$ & $58 \%$ & $\begin{array}{l}1983-2000 \\
1987-2000\end{array}$ \\
\hline $\begin{array}{l}\text { REAL COLLOBRIER } \\
\text { Rimbaud } \\
\text { Valescure } \\
\end{array}$ & $\begin{array}{l}146 \text { ha } \\
930 \text { ha }\end{array}$ & $\begin{array}{c}\text { maquis ; feu ; repousse } \\
\text { maquis }\end{array}$ & $20 \%$ & $\begin{array}{c}1966-1990 ; 1990-2000 \\
1966-2000\end{array}$ \\
\hline $\begin{array}{l}\text { MONT LOZERE } \\
\text { Latte } \\
\text { Cloutasses }\end{array}$ & $\begin{array}{l}19,5 \text { ha } \\
81 \text { ha }\end{array}$ & $\begin{array}{c}\text { épicéas ; coupe ; repousse } \\
\text { pelouse pâturée }\end{array}$ & $\begin{array}{l}20 \% \\
10 \% \\
\end{array}$ & $\begin{array}{c}1981-1987 ; 1987-1999 \\
1981-1999\end{array}$ \\
\hline
\end{tabular}

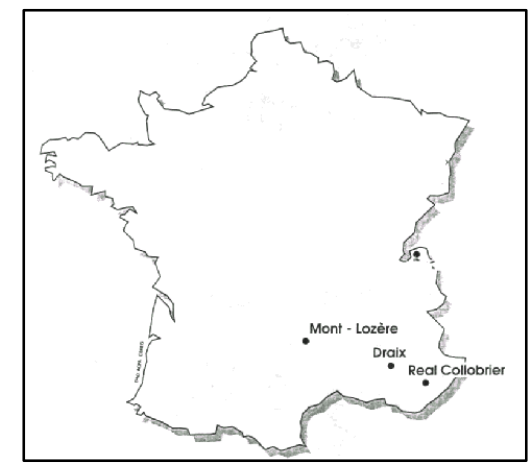

Figure 1 - Les sites de recherche en hydrologie forestière en France méditerranéenne.

\section{CONSEQUENCES DE LA FORET SUR LES CRUES}

Les résultats concernant les conséquences de la forêt sur les crues (pointe ou volume) sont très différents d'un site à l'autre.

\section{III.1 A Draix}

Le dispositif expérimental comporte un bassin reboisé à la fin du $19^{\text {ème }}$ siècle (le Brusquet) et un bassin non reboisé, dont le taux de couverture végétale est faible (Tab.1). Les pluies annuelles sont de l'ordre de $940 \mathrm{~mm}$, avec des maxima journaliers décennaux de $80 \mathrm{~mm}$, et de fortes intensités lors d'orage d'été et d'automne.

Le premier effet du couvert végétal, est l'augmentation du seuil minimal de pluie nécessaire à l'apparition d'un écoulement : les crues du bassin boisé sont moins fréquentes [1]. Pour des crues produites par des pluies analogues, les pointes de crue sont retardées d'au moins une demi-heure dans le bassin boisé.

Les pointes de crue sont très fortes pour le bassin non reboisé, où elles ont atteint $23 \mathrm{~m} / \mathrm{s} / \mathrm{km}^{2}$ lors d'un épisode pluvieux de $53 \mathrm{~mm}$. Elles sont en moyenne 5 fois plus faibles pour le bassin reboisé. Un exemple est fourni par la crue du 27 mai 1988 (Fig. 2).

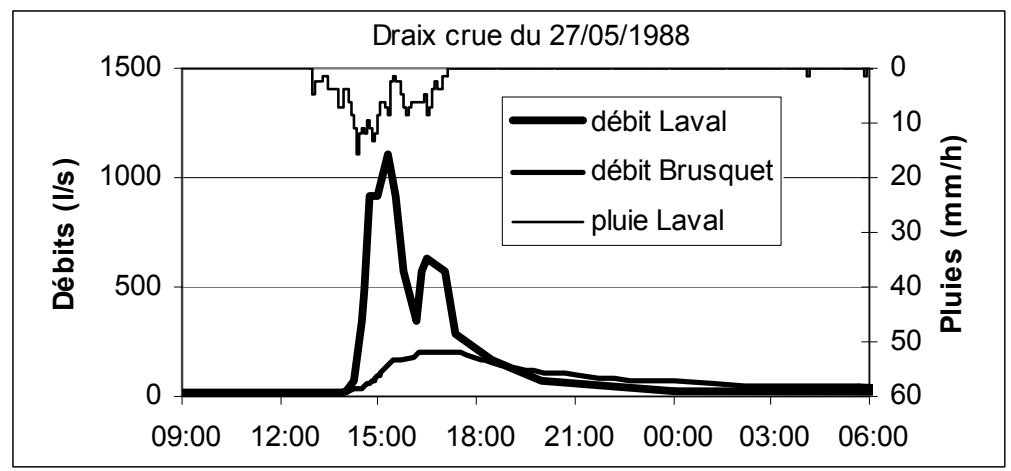

Figure 2 - La crue du 27 mai 1988 dans les bassins du Brusquet (reboisé) et du Laval. 
Une averse de $21 \mathrm{~mm}$ provoque une pointe de crue six fois plus forte dans le bassin non reboisé du Laval $\left(1,3 \mathrm{~m}^{3} / \mathrm{s} / \mathrm{km}^{2}\right)$ que dans celui reboisé du Brusquet $\left(0,2 \mathrm{~m}^{3} / \mathrm{s} / \mathrm{km}^{2}\right)$, soit dans un rapport de 1 à 6,5 .

La limitation des volumes de crue est moins importante que celle des pointes parce que la crue est plus longue dans le bassin boisé. Le rapport est généralement de 1à 2 [1].

\section{III.2 Dans les bassins du Réal Collobrier}

Les pluies annuelles sont de l'ordre de $1200 \mathrm{~mm}$, avec des pluies journalières décennales de $150 \mathrm{~mm}$, et toujours de fortes pluies d'automne.

Un incendie de forêt, durant l'été 1990 à détruit $80 \%$ environ du bassin du Rimbaud, dont les débits étaient mesurés depuis 25 ans. L'automne qui a suivi cet incendie, le comportement du ruisseau du Rimbaud est devenu fortement impulsionnel. Le débit maximal a atteint $5 \mathrm{~m} / \mathrm{s} / \mathrm{km}^{2}$, pour des précipitations ne présentant aucun caractère exceptionnel, ni par leur abondance ni par leur intensité. Les montées de crue ont souvent demandé moins de cinq minutes, et les décrues ont été elles-mêmes très rapides. Le caractère excessif pris par les réactions du cours d'eau aux précipitations s'explique par la production d'un ruissellement sur les versants mis à nu par l'incendie [2].

Une modélisation "pluie-débit" au pas de temps journalier, permet de comparer les débits observés avec ceux simulés, sur une même période, par le modèle calé sur les données recueillies avant l'incendie ([3], fig. 3).

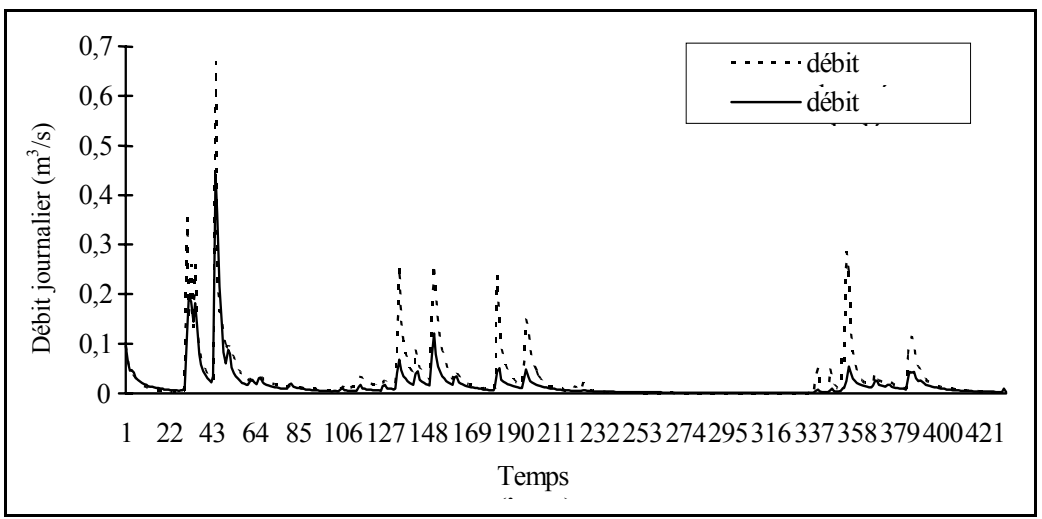

Figure 3 - Comparaison des débits journaliers mesurés après l'incendie avec les valeurs reconstituées à partir des informations recueillies avant le feu (la période représentée démarre le 26 octobre 1990 ; le temps est en jours).

Cette figure fait clairement ressortir les effets de la disparition du couvert végétal pendant les périodes pluvieuses, qui correspondent aux pluies d'automne et de printemps.

\section{III.3 Sur le Mont-Lozère}

Le dispositif expérimental se compose de deux bassins, l'un en pelouse pâturée et l'autre en épicéas. Après 6 années de suivi, une attaque parasitaire du bassin en épicéas a conduit à sa coupe à blanc. Les données obtenues sur ce bassin avant et après la coupe, sont comparées à celles du bassin témoin des Cloutasses. Les pluies sont très abondantes : les précipitations annuelles moyennes avoisinent $1950 \mathrm{~mm}$ et les abats d'eau en 24 heures peuvent atteindre $400 \mathrm{~mm}$.

Les conditions de travail très difficiles qui prévalent lors des pluies cévenoles gênent la vérification des courbes de tarage théoriques pour les débits les plus forts. D'après la comparaison entre les pointes de crue observées sur les deux bassins, le débit de pointe annuel serait plus faible dans le bassin boisé $\left(1 \mathrm{~m}^{3} / \mathrm{s} / \mathrm{km}^{2}\right)$ que dans celui en pelouse $\left(1,45 \mathrm{~m}^{3} / \mathrm{s} / \mathrm{km}^{2}\right)$, mais la tendance s'inverserait avec les débits décennaux, puisque ceux-ci s'élèveraient à $3,15 \mathrm{~m}^{3} / \mathrm{s} / \mathrm{km}^{2}$ pour le bassin forestier et à $3 \mathrm{~m} / \mathrm{s} / \mathrm{km}^{2}$ seulement pour celui en pelouse [4].

Au sujet des volumes de crue, qui sont bien mieux connus, un simple graphique de double cumul établi à partir des volumes journaliers écoulés par les bassins de la Latte (avant et après la coupe) et des Cloutasses (considéré comme témoin) lors des fortes crues est déjà informatif. A ce sujet, la figure 4 ne montre pas de cassure qui indiquerait un changement de fonctionnement du bassin de la Latte en relation avec la coupe. 


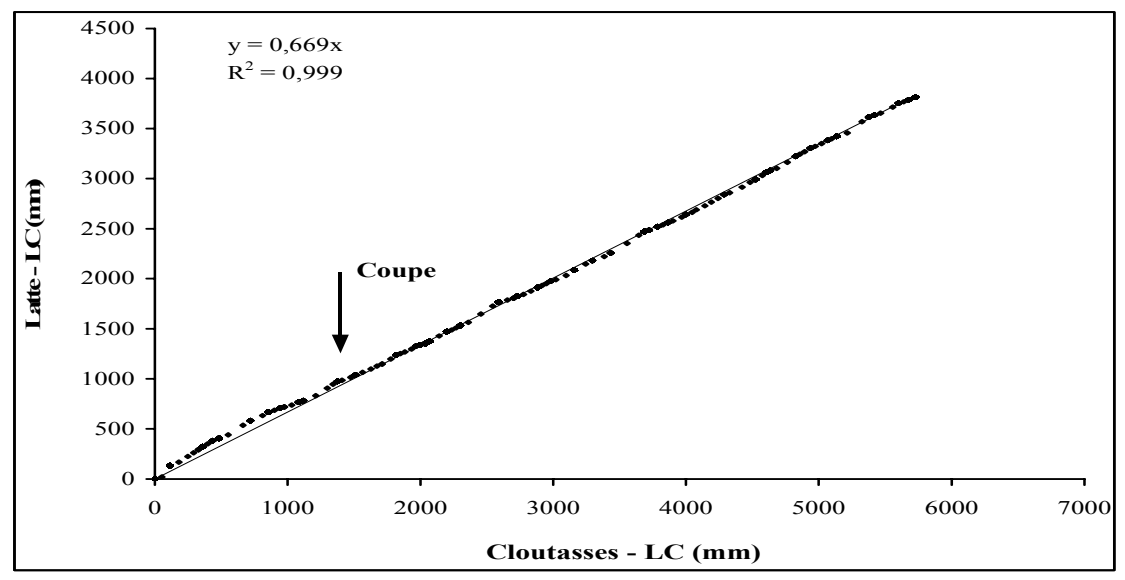

Figure 4 - Double cumul des lames d'eau écoulées par les bassins de la Latte et des Cloutasse lors des très fortes crues $-Q$ Cloutasses $>30 \mathrm{~mm} / j o u r$ (des écoulements de cette importance se produisent en moyenne moins de 8 fois par an, mais assurent plus du cinquième des écoulements (21,8\% [7]).

Ce résultat se retrouve en utilisant un seuil plus faible, puisqu'une étude du même type, menée sur des débits dépassant $20 \mathrm{~mm} /$ jour dans le bassin de la Latte, aboutit à des conclusions identiques [6] : la coupe des épicéas n'a pas entraîné d'augmentation des écoulements lors des fortes crues. Cette observation, qui serait surprenante dans d'autres conditions [2], s'explique ici par la forte perméabilité des sols et les processus spécifiques de formation des crues [7].

\section{QUE CONCLURE DU ROLE DE PROTECTION SUR LES CRUES DE LA FORET EN CLIMAT MEDITERRANEEN?}

\section{IV.1 Complexité des résultats}

A Draix, les volumes de crue du bassin forestier sont inférieurs de moitié à ceux du bassin non couvert. Les débits de pointe sont encore plus affectés, puisqu'ils sont réduits, on l'a vu, dans un rapport de 5 à 1 .

Sur le Réal Collobrier, l'incendie du bassin du Rimbaud a entraîné une augmentation très nette des débits de pointe de crue les premières années après la coupe.

Sur le Mont-Lozère, en revanche, aucune modification des débits maximaux n'a été mise clairement en évidence après la coupe pour les fortes crues.

Ainsi synthétisés, les résultats obtenus peuvent apparaître peu concordants, voire contradictoires, et amener à penser qu'il est bien difficile de conclure quant au rôle de la forêt sur les crues. En fait, ils sont complémentaires et les apparentes contradictions ne font que mettre en évidence la complexité des relations pluies-débits. Ils ne peuvent se comprendre sans prendre en compte les rôles spécifiques que jouent la nature et l'épaisseur des sols, le type de végétation (lorsque le bassin de référence n'est pas forestier), les conditions climatiques... qui déterminent les processus hydrologiques à l'origine de la forme comme du volume des crues.

\section{IV.2 Rôle de la végétation forestière sur les écoulements de crue ; considérations théoriques}

\section{IV.2.1 Processus de genèse des crues}

Les crues sont liées à une circulation rapide de l'eau sur les versants, que cette circulation soit strictement superficielle (on parle de ruissellement) ou par transmission de pression dans l'épaisseur du sol et exfiltration rapide de la nappe dans le cours d'eau [10] :

On distingue classiquement deux types de ruissellement : d'une part, le ruissellement par refus d'infiltration d'un sol non saturé (dépassement d'un seuil d'infiltration) et, d'autre part, le refus d'infiltration d'un sol saturé (dépassement d'un seuil de saturation). Cette distinction présente pourtant l'inconvénient de ne pas faire la différence entre le ruissellement dit «hortonien », lié à une très faible perméabilité intrinsèque des sols, et le ruissellement résultant de l'évolution - parfois très rapide - de l'état de surface de sols mal protégés par la végétation, sous l'impact des gouttes de pluie.

Il faut par ailleurs noter que la forêt influence les caractères hydrodynamiques des sols en agissant, de manière directe ou indirecte, sur leur structure et leur stabilité. Les sols n'évoluant que lentement, les effets d'un boisement ou d'un déboisement peuvent ne pas être immédiatement sensibles. Une déforestation, si elle ne s'accompagne pas de mise en culture ou d'érosion des sols, ne provoque donc pas forcement une aggravation 
significative des crues, surtout lorsque la revégétalisation est rapide. C'est bien ce qui s'observe sur le Mont-Lozère, il est vrai dans des conditions assez particulières : précipitations très abondantes qui minimisent le rôle de l'interception des pluies et sols extrêmement filtrants qui empêchent l'apparition du ruissellement par dépassement du seuil d'infiltration.

\section{IV.2.2 Conséquences de la végétation forestière sur les volumes de crue}

- Lorsque le ruissellement générateur des crues présente un caractère hortonien, avec des sols très peu perméables, la forêt modifie la structure du sol, améliorant ses capacités d'infiltration. Mais ces caractéristiques restent acquises indépendamment du couvert végétal, et le sol peut les conserver longtemps après déboisement. - Lorsqu'il s'agit de ruissellement généré par des évolutions des états de surface, les arbres et leurs litières ne protègent pas mieux que d'autres types de végétaux couvrant bien le sol. En revanche, si la coupe de la forêt laisse le sol à nu (ce qui n'a pas été le cas sur le Mont-Lozère) ou si l'incendie détruit complètement la végétation (ce qui a été le cas pour le Rimbaud), le sol mal protégé est soumis à une modification de son état de surface, ce qui peut favoriser le déclenchement d'un ruissellement qui ne se produisait pas avant. Le fonctionnement très impulsionnel du bassin du Rimbaud immédiatement après l'incendie pourrait s'expliquer, au moins en partie, par ce type d'évolution.

- Lorsqu'il s'agit de ruissellement par saturation à partir d'une remontée de nappe, le type de couvert n'intervient pas directement sur la progression des surfaces saturées pendant la durée des pluies. Mais le type de couvert peut jouer sur l'état des réserves hydrologiques par l'intermédiaire de l'interception, laquelle est susceptible de réduire la quantité d'eau qui s'infiltre jusqu'à la nappe.

Quel que soit le processus générateur des crues, l'interception d'une partie de la pluie incidente par la végétation forestière (interception plus importante que pour tout autre type de végétation) diminue d'autant la pluie qui arrive au sol. Mais l'interception est proportionnellement plus faible lors de fortes pluies, qui sont justement celles qui provoquent les crues : dans le cas du Mont-Lozère, l'effet de la forêt est limité pour les très fortes crues, alors qu'il semble avéré pour les écoulements plus faibles.

\section{IV.2.3 Conséquences de la végétation forestière sur les pointes de crue}

Pour un bassin versant donné, l'importance des pointes de crue dépend de la vitesse de circulation de l'eau sur les versants. Or l'on considère souvent que la végétation forestière freine le ruissellement, et accroît donc les temps de concentration. Ce ralentissement n'est pourtant pas plus efficace, sinon moins, que celui exercé par d'autres types de végétation, prairie notamment. Là encore, la différence essentielle se situe entre sol nu et sol végétalisé, plus qu'entre un type de végétation et un autre.

Lorsque les transferts d'eau résultent essentiellement de processus de sub-surface, on peut penser que les sols forestiers, généralement très perméables en raison de leur structure, peuvent favoriser les transmissions de pression et/ou permettre des circulations rapides de l'eau dans le sol. Cependant tous les auteurs ne sont pas d'accord sur ce point [11].

\section{IV.3 Rôle de la végétation forestière sur les écoulements de crue ; discussion}

Des considérations qui viennent d'être exposées, il ressort que si, dans certaines conditions, la forêt diminue les débits de pointe de crue, elle n'a pas toujours d'effet perceptible par comparaison avec un autre type de végétation en bon état et couvrant bien le sol. Il est alors facile de comprendre la diversité des résultats obtenus.

Tout d'abord, il importe de noter que le protocole expérimental n'est pas le même dans les trois bassins. Dans les cas du Mont-Lozère et du Réal Collobrier, il s'agit de déboisement, ce qui veut dire que le sol forestier initial existe, et continue de jouer un rôle hydrologique. Ce sol forestier n'existe pas pour le bassin non boisé de Draix. Enfin, un incendie de forêt n'est pas une coupe : le premier laisse le sol complètement nu et peut en outre s'accompagner de modifications de la surface du sol résultant des fortes températures; le second laisse un sol protégé par les branchages des arbres coupés, dont seuls les troncs ont été exportés. Un ruissellement superficiel, résultant de la fermeture de la surface du sol sous l'impact des gouttes de pluie, peut se produire dans le premier cas. Il n'a guère de facilités pour le faire dans le second.

Ensuite, les pluies et les crues ne sont pas les mêmes. En dépit d'abats d'eau beaucoup plus forts sur le Mont-Lozère (les maximums journaliers observés approchent $400 \mathrm{~mm}$; ils n'atteignent que $150 \mathrm{~mm}$ sur le Réal Collobrier et $80 \mathrm{~mm}$ à Draix pour des pluies décennales), les pointes de crue du bassin des Cloutasses, sous pelouse, restent bien en deçà des débits enregistrés sur les bassins déboisés du Rimbaud (Réal Collobrier) et du Laval (Draix). La raison en est que les sols très filtrants et bien protégés par la végétation ne permettent la formation d'un ruissellement que lorsqu'ils sont totalement saturés : le fonctionnement hydrologique d'un bassin sous pelouse n'est donc pas différent de celui d'un bassin forestier. Dans ces conditions, on comprend que la coupe des arbres du bassin de la Latte n'ait pas changé grand chose aux écoulements de crue.

C'est d'abord l'opposition "sol nu / sol couvert", plus que celle "forêt/autre type de végétation" qui détermine des processus hydrologiques très différents. 


\section{CONCLUSION}

Les études menées en France sur l'hydrologie forestière sous climat méditerranéen ont montré la complexité du rôle de la forêt sur les écoulements. Il est aussi faux de considérer que la forêt n'a pas d'influence sur les crues que d'affirmer qu'elle les réduit systématiquement !

La complexité des relations pluies-débits fait qu'il n'est pas possible de prévoir les conséquences d'un boisement ou d'un déboisement sans comprendre le fonctionnement hydrologique du milieu considéré. Celui-ci dépendra - entre autres - de la nature des formations superficielles, des conditions climatiques, des caractéristiques de la végétation.

Par ailleurs, il n'est pas inutile d'attirer l'attention sur le fait que les incertitudes sur les données de base (pluies et débits) à partir desquelles on raisonne sont probablement souvent du même ordre de grandeur que les effets des paramètres que l'on veut estimer : ici le rôle de la végétation. Et quant à ces incertitudes s'ajoutent les hypothèses des modèles...

Il est pourtant possible de tirer de cette synthèse un certain nombre d'enseignements :

- D'abord, l'opposition est avant tout entre sol nu et sol couvert par la végétation (quel que soit le type de végétation), bien plus qu'entre un type de couvert végétal et un autre. Si les défrichements provoquent généralement de fortes perturbations du milieu, c'est parce que la forêt est le plus souvent remplacée par des cultures, et que ces cultures laissent le sol à nu une grande partie de l'année. Les effets de l'incendie du Rimbaud sur les crues se sont atténués dès que le sol a été largement recolonisé par une végétation buissonneuse ou arbustive. Il n'y a guère de différence de fonctionnement sur le Mont-Lozère entre le bassin en pelouse et ceux en forêt. A l'opposé, le bassin très mal couvert du Laval, à Draix, présente un comportement hydrologique radicalement différent du bassin boisé du Brusquet.

- Ensuite, il faut noter l'importance du sol forestier. Son maintien après la disparition de la forêt peut expliquer les faibles conséquences de la disparition des arbres. Mais le sol forestier doit être considéré comme une création de la forêt, même s'il demeure comme une entité indépendante après la disparition de celle-ci.

Ainsi le rôle de la forêt sur les crues, s'il peut s'avérer considérable dans certaines conditions, est loin d'être toujours et partout très sensible.

\section{Bibliographie}

[1] Richard D., Mathys N., 1999 - Historique, contexte technique et scientifique des BVRE de Draix. Caractéristiques, données disponibles et principaux résultats acquis au cours des dix ans de suivi. Actes du séminaire "Les bassins versants expérimentaux de Draix, laboratoire d'étude de l'érosion en montagne" (Draix, Le Brusquet, Digne, 1997), Cemagref-Editions, coll. Actes de colloques, Mathys N. édit., pp 11-28.

[2] Martin C., Lavabre J., 1997 - Estimation de la part du ruissellement sur les versants dans les crues du ruisseau du Rimbaud (massif des Maures, Var, France) après l'incendie de forêt d'août 1990. Journal des Sciences Hydrologiques, vol. 42, $\mathrm{n}^{\circ}$ 6, pp 893-907.

[3] Lavabre J., Sempere-Torres D., Cernesson F., 1993 - Hydrological consequences of fire : changes on the hydrological response of a little Mediterranean basin a year after the fire. J. Hydrol., vol. 142, pp 273-299.

[4] Lavabre J., Martin C., avec la collaboration de Didon-Lescot J.F., 1999 - Appréciation de l'impact des coupes forestières sur l'hydrologie et l'érosion des sols. Cas de la forêt d'Altefage (commune du Pont-deMontvert - 48). Rapport à la DDAF de Lozère, Edit. Cemagref, Aix-en-Provence, $28 \mathrm{p}$.

[5] Martin C., Didon-Lescot J.F., avec la collaboration de Cosandey C., 2000 - Le rôle du couvert végétal sur le fonctionnement hydrologique des bassins versants expérimentaux du Mont-Lozère. In : Etude intégrée du bassin versant du Haut-Tarn appliquée à la gestion des ressources en eau et des fonctionnements hydrobiologiques : contribution de l'UMR "ESPACE" à la connaissance des fonctionnements hydrologiques et hydrochimiques, Rapport intermédiaire pour le Parc national des Cévennes et l'Agence de l'Eau Adour-Garonne, pp 34-53.

[6] Cosandey C., avec la collaboration de Martin C. et Didon-Lescot J.F., 2000 - Forêt et écoulements : étude des conséquences d'une coupe forestière sur le bilan d'écoulement annuel, les crues et les étiages. Rapport sectoriel pour le rapport final du contrat européen FOREX, 34 p.

[7] Cosandey C., 1994 - Formation des crues "cévenoles" dans des bassins élémentaires du Mont-Lozère. Revue des Sciences de l'Eau, vol. 7, pp 377-393.

[8] Cosandey C., Robinson M., 2000 - Hydrologie continentale. Edit. Armand Colin, 360 p.

[9] Rawlins B.G., Baird A.J., Trudgll S.T., Hornung M., 1997 - Absence of preferential flows in the percolating waters of a coniferous forest Soil. Hydrol. Process, vol. 11, pp 575-585. 